\title{
Experiencias de burnout en odontólogos de la Comunidad Valenciana
}

\author{
Javier Molina-Hernández, Lorena González García, y \\ Lucía Fernández-Estevan \\ Universidad Europea de Valencia (España)
}

Los profesionales de la salud presentan un alto nivel de burnout siendo la odontología una especialidad poco estudiada. El objetivo del presente estudio es analizar las experiencias de burnout de los odontólogos de la Comunidad Valenciana, analizando su relación con la percepción de satisfacción laboral, autoestima general y el estrés percibido. Se recogieron los datos de 366 odontólogos, que cumplimentaron una batería de cuestionarios online y de manera anónima entre enero y mayo de 2018. Los resultados mostraron que los participantes presentaban niveles de burnout por debajo de la media, un nivel de estrés ligeramente superior a la media y una buena percepción con su satisfacción laboral y nivel de autoestima. La autoestima se relacionaba positiva y significativamente con la satisfacción y negativamente con el estrés y el burnout. Finalmente, los análisis de regresiones lineares mostraron que la percepción de estrés predecía positiva y significativamente la sensación de burnout global, mientras que la percepción de satisfacción laboral y autoestima predecían negativa y significativamente el burnout global de los participantes. Concluimos que para evitar el desarrollo de burnout es necesario promover una adecuada satisfacción laboral y unos bajos niveles de estrés junto con una alta autoestima en la vida del profesional.

Palabras clave: Burnout, estrés, odontología autoestima, satisfacción laboral

Experiences of burnout in odontologists in the Valencian Community. The health professionals have high stress and burnout levels being dentistry a little-studied specialty in the health profession in this respect. The objective of this study was to analyze burnout in dentists in the Valencian Community, analyzing the relationship in dentists between job satisfaction, general self-esteem and perceived stress. To this end, an observational study was carried out, where data was collected from 366 dentists, who filled out a series of online questionnaires anonymously between January and May 2018. The results showed that the participants had burnout levels below average, stress levels slightly above average, with high levels of job satisfaction and self-esteem. Self-esteem was positively and significantly related to job satisfaction and negatively to stress and burnout. Finally, linear regression analysis showed that the perception of stress positively and significantly predicted the overall burnout sensation experienced by the participants, while both job satisfaction perception and self-esteem negatively and significantly predicted participant burnout. We concluded that to prevent burnout, adequate job satisfaction and low levels of stress must be promoted, together with high levels of self-esteem in the professional's life.

Keywords: Burnout, stress, odontology, self-esteem, job satisfaction

Correspondencia: Javier Molina Hernandez. Facultad Ciencias de la Salud Universidad Europea de Valencia. Paseo de la Alameda 7. C.P.:46010 Valencia (Spain). E-mail: javier.molina@universidadeuropea.es 
Desde que en la década de los años 80 la Organización Internación del Trabajo (OIT) y la Organización Mundial de la Salud (OMS) coincidieran que no sólo los factores químicos, físicos y/o biológicos presentes en el trabajo eran importantes para la salud de los trabajadores, se ha profundizado sobre la implicación de diferentes variables psicosociales en la salud de los empleados (Siegrist, 1990).

Los factores de riesgo psicosociales en el ámbito de la empresa recogen aquellos factores organizacionales que pueden provocar estrés en el trabajador afectando a su salud (Benavides et al., 2002).

En el ámbito sanitario los médicos y las enfermeras han sido los profesionales más estudiados (Fernández-López, Siegrist, Rödel, y Hernández-Mejía, 2003; GarcíaRodríguez, Gutiérrez-Bedmar, y Bellón-Saame, 2015; Peiró, 1999; Sobrequés et al., 2003). Sin embargo, hemos encontrado limitada literatura científica basada en la situación en la que se encuentran los odontólogos, profesionales en los que se centrará el presente estudio.

Centrándonos en la limitada literatura en el contexto odontológico (Kay y Lowe, 2008; Moore y Brødsgaard, 2001), se destaca que los factores más estresantes son por un lado los relacionados con aspectos de la clínica (p. ej., problemas de agenda, problemas con el personal, aspectos administrativos o económicos) y por otro lado los que implican la relación con los pacientes (p. ej., ansiedad, citas anuladas, o poca colaboración). Una situación de estrés laboral crónico puede desencadenar en el síndrome de burnout afectando en mayor o menor medida a diferentes colectivos profesionales. Algunos de los autores que más han contribuido al estudio del burnout son Maslach y su equipo de colaboradores (Maslach y Jackson, 1981), quienes defienden que el síndrome de degaste profesional se caracteriza por los sentimientos que presenta un trabajador respecto a su actividad laboral de agotamiento emocional, despersonalización y falta de realización personal.

Según la literatura, en Europa podemos encontrar niveles altos de burnout entre profesionales de la odontología, concretamente un $26 \%$ de dentistas en Irlanda (Gorter y Freeman, 2011), un 18.2\% de dentistas en Reino Unido (Denton, Newton, y Bower, 2008) y un 20\% en Lituania (Puriene, Aleksejuniene, Petrauskiene, Balciuniene, y Janulyte, 2008). En España hemos encontrado dos estudios centrados en las experiencias de burnout de los odontólogos. Uno se realizó sobre dentistas generales evaluando a 1913 dentistas españoles, y se vio que un $6 \%$ de ellos presentaban niveles muy altos de burnout y un $29 \%$ niveles altos, siendo mayor el nivel de agotamiento emocional en el sur de España que en el norte (Aguilar, 2015). En otro estudio realizado sólo sobre periodoncistas, se vio que estos sufrían burnout en el $13.4 \%$ de los casos (Reyes-Torres, Ríos-Santos, López-Jiménez, Herrero-Climent, y Bullón, 2012).

En las dos últimas décadas la Odontología española ha experimentado un crecimiento descontrolado del número de profesionales, incrementándose cerca de un 
102\% (Llodra-Calvo, 2010). Este hecho junto con el hecho de enfrentarse a situaciones de precariedad laboral que dificultan el correcto desarrollo las funciones laborales (Pinilla-Domínguez, 2012; Rivera, 2015) conduce a la insatisfacción laboral (Luzzi y Spencer, 2011).

Estudios previos defienden que el nivel de satisfacción laboral puede verse afectado por factores como el sexo, la edad, las horas trabajadas, o si trabaja en el sector público o privado así como su sueldo (Goetz, Campbell, y Broge, 2012).

Teniendo en cuenta esta situación, el objetivo de nuestro estudio se centra en analizar las experiencias de burnout en los odontólogos, estudiando cómo el estrés percibido en el entorno laboral, la satisfacción que experimentan hacia su trabajo y la autoestima general pueden influir sobre sus sentimientos de burnout. Para alcanzar el objetivo propuesto se presentan las siguientes hipótesis: 1) el nivel de burnout y estrés percibido será elevado; 2) el nivel de autoestima y de satisfacción laboral de los odontólogos encuestados será elevado; 3) el burnout percibido por los odontólogos se relacionará positivamente con su estrés; 4) el burnout percibido por los odontólogos se relacionará negativamente con sus experiencias de autoestima y de satisfacción laboral; 5) el estrés, predecirá positivamente la percepción de burnout de los odontólogos mientras que la satisfacción laboral y la autoestima lo harán de manera negativa.

\section{MÉTODO}

\section{Participantes}

Los participantes del estudio fueron 366 colegiados en la Comunidad Valenciana (provincias de Alicante, Castellón y Valencia) contactados a través de los colegios oficiales de odontólogos de cada provincia.

El $71 \%(n=260)$ eran mujeres $(71 \%)$ y el $29 \%(n=106)$ eran hombres. La edad de los participantes oscilaba entre los 24 a los 69 años $(M=37.63 ; D T=9.69)$. Los criterios de inclusión fueron: 1) ser licenciado o graduado en la titulación de odontología y 2) estar colegiado en los colegios oficiales de odontólogos de las provincias de la Comunidad Valenciana. Al indicar las especialidades de trabajo, los participantes informaron que el $26.8 \%$ de ellos trabajaba en cirugía e implantes, el 13.9\% trabajaba en periodoncia, el $15.3 \%$ trabajaba en ortodoncia, el $9.8 \%$ trabajaba en odontopediatría, el $21.9 \%$ trabajaba en endodoncia. Un $61.5 \%$ afirmaba trabajar también como odontólogo general. Además, el 38\% también participaban en tareas docentes. En relación con su situación fiscal, el $36.3 \%$ de los participantes trabajaba como autónomo en su propia clínica, el 30.3\% trabajaba como autónomo para otras clínicas, el 17.2\% compaginaba el trabajo como autónomo en su propia clínica con el trabajo en otras clínicas, y el 16.1\% refería ser contratado. En cuanto al tiempo trabajado, el $37.7 \%$ de los encuestados refería trabajar entre 30 y 40 horas semanales, el 35.2\% trabajaban más de 40 horas a la semana, 
el $20.8 \%$ trabajaba entre 20 y 30 horas a la semana y el $6.3 \%$ trabajaba menos de 20 horas a la semana.

\section{Instrumentos}

Datos sociodemográficos. Se presentaron un total de ocho preguntas elaboradas ad hoc para obtener información sobre el sexo, práctica odontológica más habitual, tipo de contrato, y horas trabajadas. La elaboración de dichas preguntas se basó en estudios previos que realizaron preguntas similares (p. ej., Goetz, Hasse, Szecsenyi, y Campbell, 2016). En este apartado los participantes encontraban preguntas de respuesta abierta como “¿Cuántos años lleva ejerciendo la odontología?” o de respuesta cerrada como “¿Qué práctica odontológica realizan con mayor frecuencia?" que debían responder eligiendo entre cinco opciones (i.e., periodoncia, ortodoncia, cirugía, odontología general o endodoncia).

Burnout. Para evaluar la experiencia de burnout de los participantes se utilizó la versión española (Seisdedos, 1997) del Inventario de Burnout de Maslach (Hobroll y Freedy, 1993). Este cuestionario evalúa el desgaste profesional, y los sentimientos y la actitud del profesional hacia los pacientes y hacia su propio trabajo. Está compuesto por 22 ítems que se contestan a través de una escala de tipo Likert de 7 puntos que van desde 1 "nunca" a 7 "todos los días". La escala se divide en 3 subescalas: agotamiento emocional, despersonalización y desrealización personal. El agotamiento emocional se evalúa a través de 9 ítems como "Me siento emocionalmente agotado por mi trabajo", la despersonalización se evalúa a través de 5 ítems como "Me he vuelto más insensible con la gente desde que ejerzo mi profesión", y la desrealización personal con 8 ítems como "Siento que los pacientes me culpan por algunos de sus problemas". La fiabilidad y validez de la escala ha sido probada por múltiples estudios tanto en el ámbito odontológico (Ahola y Hakanen, 2007), como en otros campos de la salud (Hakanen, Schaufeli, y Ahola, 2008).

Satisfacción laboral. La satisfacción laboral general se evaluó con la escala de satisfacción laboral del Warr, Cook, y Wall (1979) en su versión española (Pérez y Fidalgo, 1993). Este instrumento está compuesto por 15 ítems que se evalúan por medio de una escala de tipo Likert de 7 puntos, donde 1 es "muy insatisfecho" y 7 "muy satisfecho". A su vez esta escala se divide en dos subescalas para medir factores intrínsecos y extrínsecos. Los factores intrínsecos se miden con 7 ítems como “¿Cómo se siente usted con respecto a la libertad para elegir su propio método de trabajo?”. Los factores extrínsecos se miden a través de 8 ítems como “¿Cómo se siente usted con respecto a las condiciones físicas de su trabajo?". La validez y la fiabilidad de esta escala fue demostrada en estudios en diferentes ámbitos laborales, incluyendo el odontológico (Arias, Rivera, y Ceballos, 2017; Goetz et al., 2012). 
Estrés percibido. La evaluación del estrés se realizó mediante la versión española (Remor, 2006) de la Escala de estrés percibido (Cohen, Kamarck, y Mermelstein, 1983). Esta escala consta de 14 ítems con un formato de respuesta de tipo Likert de 5 puntos, siendo 1 "nunca" y 5 "muy a menudo" y recoge las experiencias de estrés sufridas durante los últimos 30 días. Dentro de la escala podemos encontrar preguntas como "En el último mes, ¿con qué frecuencia se ha sentido incapaz de controlar las cosas importantes en su vida?". La validez y fiabilidad de esta escala fue demostrada en múltiples estudios tanto en odontología como en otras ramas de sanidad (Chakraborti et al., 2013; Mahmoud y Ghada, 2012).

Autoestima. Para valorar la percepción de autoestima global que tiene una persona se utilizó la Escala de Autoestima de Rosenberg (Rosenberg, 1965) en su versión española (Atienza, Moreno, y Balaguer, 2000). Este instrumento es de naturaleza unidimensional y consta de 10 ítems que se contestan a través de una escala de tipo Likert de 4 puntos, siendo 1 "totalmente en desacuerdo" hasta 4 "totalmente de acuerdo". La escala incluye ítems como "Me gustaría poder sentir más respeto por mí mismo". La fiabilidad y validez de este instrumento ha sido probada en diferentes ámbitos laborales (Benyamini, Leventhal, y Leventhal, 2004).

\section{Procedimiento}

El estudio fue aprobado por el comité ético de la Universidad Europea de Madrid.

La recogida de información se realizó entre enero y mayo de 2018 mediante la cumplimentación de un paquete de cuestionarios enviados a través de la página web de Survey Monkey. Antes del cuestionario se presentó un consentimiento informado en el cuál se informaba que la participación en el estudio era voluntaria, anónima, y que podían abandonar el estudio cuando lo deseasen. Esta parte era de obligado cumplimiento para poder continuar con el cuestionario. La configuración no permitía repetir el cuestionario por el mismo participante más de una vez. Todas las preguntas estaban configuradas para ser obligatoriamente respondidas, de lo contrario no podían pasar de página.

\section{RESULTADOS}

\section{Análisis preliminares}

Los resultados sobre los estadísticos descriptivos y los índices de fiabilidad se pueden observar en la tabla 1. En relación con los índices de fiabilidad de las escalas utilizadas, los resultados indicaron que los instrumentos utilizados en este estudio presentaron una fiabilidad adecuada $(\alpha=.65-.93)$, existiendo solo una escala con un coeficiente de fiabilidad por debajo de .70 . 
Los resultados descriptivos del estudio informaron que los participantes del estudio referían tener un cansancio emocional ligeramente por encima de la media del cuestionario $(M=3.698 ; D=3.698)$, mientras que presentaron unos valores relativamente bajos en su percepción de despersonalización $(M=2.112 ; D T=1.028)$ y de desrealización personal $(M=1.764 ; D T=.772)$. Considerando el burnout en su conjunto, los odontólogos que participaron en el estudio presentaron unas puntuaciones por debajo de la media de los cuestionarios $(M=2.642 ; D T=.851)$. Si nos centramos en la percepción de satisfacción laboral, los odontólogos y estomatólogos que participaron en nuestro estudio informaron estar altamente satisfechos con su trabajo $(M=5.177 ; D T=1.072)$, así como percibir en elevado nivel de autoestima $(M=3.320$; $D T=.430$ ). Por otro lado, podemos observar cómo los participantes referían tener unos niveles de estrés medios y ligeramente por encima de la media del cuestionario $(M=2.598 ; D T=.581)$.

Tabla 1. Estadísticos descriptivos de las variables estudiadas

\begin{tabular}{lcccc}
\hline \multicolumn{1}{c}{ Variable } & Rango respuesta & $M$ & $D T$ & Alpha \\
\hline Cansancio emocional & $1-7$ & 3.698 & 3.698 & 0.887 \\
\hline Despersonalización & $1-7$ & 2.112 & 1.028 & 0.649 \\
\hline Desrealización personal & $1-7$ & 1.764 & .772 & 0.827 \\
\hline Burnout global & $1-7$ & 2.642 & .851 & 0.879 \\
\hline Satisfacción laboral & $1-7$ & 5.177 & 1.072 & 0.932 \\
\hline Estrés & $1-5$ & 2.598 & .581 & 0.892 \\
\hline Autoestima & $1-4$ & 3.320 & .430 & 0.821 \\
\hline
\end{tabular}

Relación entre las variables de estudio

Los análisis de correlaciones parciales (Tabla 2) mostraron que el cansancio emocional se relacionaba positiva y significativamente con las otras dos dimensiones del burnout, la despersonalización $(r=.416 ; p=.000)$ y la desrealización personal $(r=347$. $P=.000)$. En la misma dirección, esta variable mostró una relación positiva y significativa con la percepción de estrés $(r=.610, p=.000)$, mientras que mostró una relación negativa y significativa con la satisfacción laboral $(r=-.458 ; p=.000)$ y con la autoestima $(r=-.387 ; p=.000)$. La despersonalización mostró una relación positiva y significativa con la desrealización $(r=.407 ; p=.000)$ y con la percepción de estrés ( $r=.351 ; p=.000)$, y mostró una relación negativa y significativa con la satisfacción laboral $(r=-.310 ; p=.000)$ y con la autoestima $(r=-.380 ; p=.000)$. La satisfacción laboral se relacionaba de manera negativa y significativa con el estrés $(r=-.490$; $p=.000)$ y de forma positiva y significativa con la autoestima $(r=.476 ; p=.000)$. Por último, la relación entre el estrés y la autoestima resultó ser negativa y significativa $(r=-.512 ; p=.000)$. 
Tabla 2. Correlaciones entre las variables estudiadas

\begin{tabular}{lcccccc}
\hline \multicolumn{1}{c}{ Variable } & 1 & 2 & 3 & 4 & 5 & 6 \\
\hline 1. Cansancio emocional & \multicolumn{7}{c}{. } \\
\hline 2. Despersonalización & $.416^{* * *}$ & & & & & \\
\hline 3. Desrealización personal & $.347^{* * *}$ & $.407^{* * *}$ & & & & \\
\hline 5. Satisfacción laboral & $-.458^{* * *}$ & $-.310^{* * *}$ & $-.428^{* * *}$ & $-.512^{* * *}$ & & \\
\hline 6. Estrés & $.610^{* * *}$ & $.351^{* * *}$ & $.359^{* * *}$ & $.595^{* * *}$ & $-.490^{* * * *}$ & \\
\hline 7. Autoestima & $-.387^{* * *}$ & $-.380^{* * *}$ & $-.465^{* * *}$ & $-.512^{* * *}$ & $.476^{* * *}$ & $-.544^{* * *}$ \\
\hline Nota: ${ }^{* * *} p<.001$ & & & & & &
\end{tabular}

Tal como puede observarse en la tabla 3, los análisis de regresiones lineares mostraron que la percepción de estrés que tenían los odontólogos predecía positiva y significativamente la sensación de burnout global que experimentaban los participantes $(\beta=.373 ; p=.000)$, mientras que la percepción de satisfacción laboral $(\beta=-.235$; $p=.000)$ y la autoestima $(\beta=-.197 ; p=.000)$ predijeron negativa y significativamente el burnout global de los participantes. En su conjunto, el estrés, la satisfacción laboral y la autoestima predecían un $44,3 \%$ de la variabilidad de la percepción de burnout global $\left(R^{2}=.443\right)$.

Tabla 3. Predicción del burnout global por la percepción de estrés y la satisfacción laboral

\begin{tabular}{lcc}
\hline & \multicolumn{2}{c}{ Burnout } \\
\hline Predictor & B. & ES \\
\hline 1.Estrés & .373 & $.072^{* * *}$ \\
\hline 2.Satisfacción laboral & -.235 & $.037^{* * *}$ \\
\hline 3. Autoestima & -.197 & $.097 * *$ \\
\hline Nota: $* * p<.01 ; * * p<.001$. & &
\end{tabular}

\section{DISCUSIÓN Y CONCLUSIONES}

El presente estudio describe el nivel de burnout de los odontólogos de la Comunidad Valenciana, analizando la influencia del estrés, la autoestima y la satisfacción laboral. En general, los resultados obtenidos apoyan las hipótesis planteadas, destacando que los altos niveles de autoestima y satisfacción laboral contribuyen en la prevención del burnout de los odontólogos, mientras que el estrés percibido lo promueve.

La primera hipótesis del estudio defendía que el nivel de burnout y de estrés de los odontólogos encuestados sería elevado, pero los resultados muestran que tienen un nivel medio de burnout, siendo la dimensión del cansancio emocional la que presenta valores más altos. Aunque estos resultados concuerdan con otros encontrados sobre periodoncistas españoles (Ríos-Santos, Reyes-Torres, López-Jiménez, MorilloVelázquez, y Bullón, 2010), o sobre odontólogos generales (Luzzi, Spencer, Jones, y 
Teuser, 2005), esperábamos encontrar niveles de burnout más elevados tal y como habíamos observado en otros trabajos (Osborne y Croucher, 1994; Puriene et al., 2008). Normalmente los cirujanos son los que más burnout refieren y los ortodoncistas los que menos (Rada y Johnson-Leong, 2004), y dado que en nuestro estudio sólo había un $26.8 \%$ de cirujanos, el índice de burnout podría haberse visto reducido. Por otro lado, hemos encontrado niveles más altos de estrés, lo cual estaría en concordancia con la literatura revisada (Moore y Brødsgaard, 2001).

La segunda hipótesis del estudio defendía que los odontólogos presentarían unos niveles elevados de autoestima y satisfacción laboral. Esta hipótesis ha quedado confirmada por los resultados obtenidos corroborando así estudios similares (Puriene et al., 2008; Uribe, Jocelyn, y Castro-Caro, 2014). Según la literatura científica en el ámbito de la odontología, la satisfacción laboral es más alta en aquellos profesionales que trabajan por cuenta propia debido a una mayor capacidad de gestionar sus agendas, calidad de materiales y número de pacientes atendidos (Luzzi y Spencer, 2011). En nuestro estudio un $83,8 \%$ trabajaba como autónomos, lo que podría ayudar a comprender los resultados a pesar de que un alto porcentaje trabaje tanto para su clínica como para la clínica de otros.

Los resultados obtenidos en nuestro estudio confirman la tercera hipótesis presentada, en la que se defendía que las experiencias de burnout y de estrés se relacionarían positivamente entre ellas. Efectivamente, los resultados muestran que existe una relación positiva entre el estrés y el burnout, de la misma manera que se ha encontrado en estudios previos (Gorter, Albrecht, Hoogstraten, y Eijkman, 1998; Osborne y Croucher, 1994).

En línea con la cuarta hipótesis se ha observado que el burnout se relaciona de manera negativa con las experiencias de autoestima y satisfacción laboral. Esto está en línea con estudios en diferentes profesiones sanitarias (Janssen, Schaufeli, y Houkes, 1999), incluyendo la odontología (Goetz et al., 2012). Sin embargo, también existen estudios en odontología donde se han descrito niveles bajos de satisfacción laboral que se han relacionado con elevados niveles de burnout (Osborne y Croucher, 1994), y otros donde a pesar de tener un $90 \%$ de satisfacción laboral, presentaban valores elevados de burnout (Puriene et al., 2008). Todo esto resalta la importancia de seguir analizando estas relaciones en futuros estudios para poder entender los factores que puedan influir en estos hallazgos discrepantes.

La quinta hipótesis planteada en el estudio queda confirmada tras observar que el estrés, la satisfacción laboral y la autoestima predicen la percepción de burnout de los odontólogos en un 44.3\%. Tal y como se observa en estudios previos (DiMatteo, Shugars, y Hays, 1993; Janssen et al., 1999), cuando hay un nivel alto de estrés, el dentista entra en una espiral negativa y percibe como un estresor laboral al paciente problemático, ansioso o insatisfecho incluso pacientes que llegan tarde. El nivel elevado 
de estrés puede llevar a una apatía y una pobre comunicación y así como una visión mala de sí mismo, lo que al final conduce a una insatisfacción laboral, promoviendo un síndrome de burnout crónico.

Observamos algunas limitaciones en el presente estudio que consideramos importante resaltar. Por un lado, los datos analizados corresponden a los odontólogos que aceptaron contestar el cuestionario, pudiéndose entender que un odontólogo que está contento con su profesión también podría estar más dispuesto a compartir su sentimiento con otras personas. Por otro lado, el acceso al grupo de estudio ha sido limitado por dos razones 1) al haber hecho el cuestionario on-line puede que los rangos de mayor edad hayan desconfiado del enlace facilitado y 2) que existan muchos profesionales que no revisen el correo procedente de su colegio oficial. También es importante destacar que es difícil comparar resultados con estudios de otros países, donde los sistemas sanitarios, horarios de trabajo y estilos de vida difieren tanto de un sitio a otro.

Considerando las implicaciones prácticas del presente estudio, podemos resaltar que la información obtenida nos ayuda a entender mejor el ámbito laboral odontológico, así como a enfatizar la importancia que tiene presentar unos niveles altos de autoestima y satisfacción laboral junto con un nivel bajo de estrés, para poder reducir los niveles burnout en el trabajo. Estos datos podrían resultar de especial relevancia para la elaboración de estrategias de prevención de diversas psicopatologías, así como para la promoción de ambientes laborales más adaptativos.

\section{REFERENCIAS}

Aguilar, C. A. (2015). El síndrome de burnout en los dentistas en España (Tesis Doctoral). Universidad de Salamanca, Salamanca.

Ahola, K., y Hakanen, J. (2007). Job strain, burnout, and depressive symptoms: A prospective study among dentists. Journal of Affective Disorders, 104(1-3), 103-110. doi:10.1016/j.jad.2007.03.004

Arias, W., Rivera, R., y Ceballos, K. (2017). Análisis Psicométrico de la Escala de Satisfacción Laboral de Warr, Cook y Wall en una muestra multiocupacional de Arequipa, Perú. Interacciones, 3(2), 79-85. doi:10.24016/2017.v3n2.74

Atienza, F., Moreno, Y., Balaguer, I. (2000). Análisis de la dimensionalidad de la Escala de Autoestima de Rosenberg en una muestra de adolescentes valencianos. Revista de Psicología. Universitas Tarraconensis, 22, 29-42.

Benavides, F.G., Gimeno, D., Benach, J., Martínez, J.M., Jarque, S., Berra, A., y Devesa, J. (2002). Descripción de los factores de riesgo psicosocial en cuatro empresas. Gaceta Sanitaria, 16(3), 222-229. doi:10.1016/S0213-9111(02)71665-8

Benyamini, Y., Leventhal, H., y Leventhal, E. (2004). Self-rated oral health as an independent predictor of self-rated general health, self-esteem and life satisfaction. Social Science $y$ Medicine, 59, 1109-1116. doi:10.1016/j.socscimed.2003.12.021

Cohen, S., Kamarck, T., y Mermelstein, R. (1983). A global measure of perceived stress. Journal of Health and Social Behavior, 24(4), 385-396. doi:10.2307/2136404 
Chakraborti, A., Ray, P., Sanyal, D., Thakurta, R.G., Bhattacharayya, A.K., Mallick, A.K.,... y Ali, S.N. (2013). Assessing perceived stress in medical personnel: In search of an appropriate scale for the Bengali population. Indian Journal of Psychological Medicine, 35(1), 29-33. doi:10.4103/0253-7176.112197

Denton, D.A., Newton, J.T., y Bower, E.J. (2008). Occupational burnout and work engagement: A national survey of dentists in the United Kingdom. British Dental Journal, 205(7), 10 11. doi:10.1038/sj.bdj.2008.654

DiMatteo, M.R., Shugars, D.A., y Hays, R.D. (1993). Occupational stress, life stress and mental health among dentists. Journal of Occupational and Organizational Psychology, 66(2), 153-162. doi:10.1111/j.2044-8325.1993.tb00524.x

Fernández-López, J., Siegrist, J., Rödel, A., y Hernández-Mejía, R. (2003). El estrés laboral: Un nuevo factor de riesgo. ¿Qué sabemos y qué podemos hacer? Atención Primaria, 31(8), 524-526. doi:10.1157/13047736

García-Rodríguez, A., Gutiérrez-Bedmar, M., y Bellón-Saame, J.A. (2015). Atención primaria, entorno psicosocial y estrés en trabajadores sanitarios de la sanidad pública: Diferencias entre atención primaria y hospitalaria. Atención Primaria, 47(6), 359-366. doi:10.1016/j.aprim.2014.09.003

Goetz, K., Campbell, S., y Broge, B. (2012). The impact of intrinsic and extrinsic factors on the job satisfaction of dentists. Community Dentistry and Oral Epidemiology, 40(2), 474480. doi:10.1111/j.1600-0528.2012.00693.x

Goetz, K., Hasse, P., Szecsenyi, J., y Campbell, S.M. (2016). Questionnaire for measuring organisational attributes in dental-care practices: Psychometric properties and test retest reliability. International Dental Journal, 66, 93-98. doi:10.1111/idj.12209

Gorter, R.C., Albrecht, G., Hoogstraten, J., y Eijkman, M.A.J. (1998). Work place characteristics, work stress and burnout among Dutch dentists. European Journal of Oral Sciences, 106(6), 999-1005.

Gorter, R.C., y Freeman, R. (2011). Burnout and engagement in relation with job demands and resources among dental staff in Northern Ireland. Community Dentistry and Oral Epidemiology, 39(1), 87-95. doi:10.1111/j.1600-0528.2010.00568.x

Hakanen, J. J., Schaufeli, W. B., y Ahola, K. (2008). The job demands-resources model: A threeyear cross-lagged study of burnout, depression, commitment, and work engagement. Work and Stress, 22(3), 224-241. doi:10.1080/02678370802379432

Hobroll, S., y Freedy, J. (1993). Conservation of resources: A general stress theory applied to burnout. In W. Schaufeli, C. Maslach, y T. Marek (Eds.), Profesional Burnout: Recent Developments in Theory and Research (pp. 115-1255). Washington, DC: Taylor \& Francis.

Janssen, P.P.M., Schaufeli, W.B., y Houkes, I. (1999). Work-related and individual determinants of the three burnout dimensions. Work and Stress, 13(1), 74-86. doi:10.1080/026783799296200

Kay, E.J., y Lowe, J.C. (2008). Summary of a survey of stress levels, self-perceived health and health-related behaviours of UK dental practitioners in 2005. British Dental Journal, 204(11), 622-623. doi:10.1038/sj.bdj.2008.508

Luzzi, L., Spencer, A., Jones, K., y Teuser, D. (2005). Job satisfaction of dental practitioners. British Dental Journal, 50(3), 179-185.

Luzzi, L., y Spencer, A. (2011). Job satisfaction of the oral health labour force in Australia. Australian Dental Journal, 56(1), 23-32. doi:10.1111/j.1834-7819.2010.01282.x

Llodra-Calvo, J. (2010). La demografía de los dentistas en España. Situación pasada, presente y futura. Análisis 1994-2020. Madrid: Organización Colegial de Dentistas en España. 
Mahmoud, T., y Ghada, M. (2012). Application of the perceived stress scale in health care studies. An analysis of literature. International Journal of Academic Research, 4(4), 45-50.

Maslach, C., y Jackson, S.E. (1981). The measurement of experienced burnout. Journal of Occupational Behaviour, 2, 99-113. doi:10.1002/job.4030020205

Moore, R., y Brødsgaard, I. (2001). Dentists' perceived stress and its relation to perceptions about anxious patients. Community Dentistry and Oral Epidemiology, 29(1), 73-80. doi:10.1034/j.1600-0528.2001.029001073.x

Osborne, D., y Croucher, R. (1994). Levels of burnout in general dental practitioners in the southeast of England. British Dental Journal, 177(10), 372-377. doi:10.1038/sj.bdj.4808616

Peiró, J. (1999). Desencadenantes en el estrés laboral. Madrid: Ediciones Pirámide.

Pérez, J., y Fidalgo, M. (1993). Satisfacción laboral: Escala General de Satisfacción. Madrid: Instituto Nacional de Seguridad e Higiene en el Trabajo.

Pinilla-Domínguez, J. (2012). Futuro incierto de la profesión de dentista en España. Gaceta Sanitaria, 26(6), 495-496. doi:10.1016/j.gaceta.2012.06.003

Puriene, A., Aleksejuniene, J., Petrauskiene, J., Balciuniene, I., y Janulyte, V. (2008). Selfperceived mental health and job satisfaction among Lithuanian dentists. Industrial Health, 46, 247-252.

Rada, R.E., y Johnson-Leong, C. (2004). Stress, burnout, anxiety and depression among dentists. Journal of the American Dental Association, 135(6), 788-794. doi:10.14219/jada.archive.2004.0279

Remor, E. (2006). Psychometric Properties of a European Spanish version of the Perceived Stress Scale (PSS). The Spanish Journal of Psychology, 9(1), 86-93.

Reyes-Torres, M., Ríos-Santos, J.V., López-Jiménez, A., Herrero-Climent, M., y Bullón, P. (2012). Job satisfaction and depression in the Spanish Society of Periodontology and Research (SEPA) members, and their relation to the burnout syndrome. Creation of a structural model. Medicina Oral, Patología Oral y Cirugía Bucal, 17(5), 821-824. doi:10.4317/medoral.17478

Ríos-Santos, J., Reyes-Torres, M., López-Jiménez, A., Morillo-Velázquez, J.M., y Bullón, P. (2010). Burnout and depression among Spanish periodontology practitioners. Medicina Oral Patología Oral y Cirugía Bucal, 15(5), 813-819. doi:10.4317/medoral.15.e813.

Rivera, M.I. (2015). Demografía de la profesión dental en la provincia de Valencia (Tesis Doctoral). Universitat de València, Valencia.

Rosenberg, M. (1965). Society and the adolescent self-Image. Science, 148(3671), 804. doi:10.1126/science.148.3671.804.

Seisdedos, N. (1997). MBI. Inventario de "Burnout" de Maslach: Manual. Madrid: TEA.

Siegrist, J. (1990). Measuring the social dimension of subjective health in chronic illness. Psychoterapy and Psychosomatics, 54, 90-98. doi:10.1159/000288383

Sobrequés, J., Cebrià, J., Segura, J., Rodríguez, C., García, M., y Juncosa, S. (2003). La satisfacción laboral y el desgaste profesional de los médicos de atención primaria. Atención Primaria, 31(4), 227-233. doi:10.1016/S0212-6567(03)79164-1

Uribe, S., Jocelyn, O., y Castro-Caro, V. (2014). Factores que explican la satisfacción laboral de odontólogos en Chile. Revista Clínica de Periodoncia, Implantología y Rehabilitación Oral, 7(3), 128-135. doi:10.1016/j.piro.2014.07.001

Warr, P., Cook, J., y Wall, T. (1979). Scales for the measurement of some work attitudes and aspects of psychological well-being. Journal of Occupational Psychology, 52, 129-148.

Recibido: 1 de mayo de 2019

Recepción Modificaciones: 22 de mayo de 2019

Aceptado: 10 de junio de 2019 